\title{
Serum LOX-1, ADMA, and hs-CRP as Biomarkers of Ischemic Heart Disease in Chronic Kidney Disease Patients
}

\author{
Ahmed k Harfoosh ${ }^{1}$, Youssef Nassar ${ }^{2 *}$, Ahmed F Abd El Aziz ${ }^{3}$, Asmaa Saleh ${ }^{4}$, Noura M Mohamed ${ }^{5}$
}

*Corresponding Author

Youssef Nassar

yussifnassar@gmail.com

Received for publication March 15,

2020; Accepted July 5, 2020;

Published online July 5, 2020

Copyright 2020 The Authors published by Al-Azhar University, Faculty of Medicine, Cairo, Egypt. All rights reserved. This an open-access article distributed under the legal terms, where it is permissible to download and share the work provided it is properly cited. The work cannot be changed in any way or used commercially.

doi: 10.21608/aimj.2020.24355.1149

${ }^{1}$ Department of Cardiology, faculty of Medicine, Al-Azhar University, Cairo, Egypt

2 Department of Internal Medicine, Faculty of Medicine, Al-Azhar University, Cairo, Egypt

${ }^{3}$ Department of Clinical Pathology, Faculty of Medicine, Al-Azhar University, Cairo, Egypt

${ }^{4}$ Department of Biochemistry, Faculty of Pharmacy, Al-Azhar University, Cairo, Egypt

${ }^{5}$ Department of Medical Biochemistry, Faculty of Medicine, Zagazig

University, Sharkia, Egypt

\begin{abstract}
Background: Ischemic heart disease (IHD) is the main cause of death in chronic kidney disease (CKD) patients with coronary atherosclerosis, which could be predicted by inflammatory biomarkers, as highly sensitive C-reactive protein (hs-CRP). IHD is linked to other independent risk factors as asymmetric dimethylarginine (ADMA) and lectin-like oxidized LDL receptor$1(\mathrm{LOX}-1)$, which have proven roles in atherogenesis.

Objective: To evaluate the clinical significance of ADMA and LOX-1 in addition to hs-CRP as biomarkers of IHD in end-stage renal disease (ESRD) patients with regular haemodialysis.

Subjects and Methods: Patients group included 27 patients under haemodialysis with known IHD (Subgroup Ia), 13 patients under haemodialysis without IHD (Subgroup Ib), 19 patients with CKD under conservative treatment (Subgroup Ic) and a control group of 29 healthy volunteers (group II). Serum hs-CRP, ADMA, and LOX1 were assayed.

Results: The value of hs-CRP, ADMA, and LOX-1 showed a statistically significant increase in patients' group versus controls. hs-CRP and LOX-1 showed a statistically significant increase in subgroup Ia versus subgroup $\mathrm{Ib}$, also between subgroup $\mathrm{Ib}$ and subgroup Ic. Furthermore, ADMA showed a statistically significant difference between subgroup Ia and subgroup Ic, Correlation studies in subgroup Ia between hs-CRP, ADMA and LOX-1 revealed statistically significant positive correlations.

Conclusion: Serum LOX-1 is more reliable than serum ADMA in differentiating ESRD with IHD from those without IHD and its use beside hs-CRP for differentiating ESRD patients adds more strength and specification to its known predictive value.
\end{abstract}

Keywords: End-Stage Renal Disease; Ischemic Heart Disease; Asymmetric Dimethylarginine; Lectin-Like Oxidized LDL Receptor-1

Disclosure: The authors have no financial interest to declare in relation to the content of this article. The Article Processing Charge was paid for by the authors.

Authorship: All authors have a substantial contribution to the article

\section{INTRODUCTION}

Chronic renal disease is a worldwide major health problem, with increasing incidence, prevalence, high costs, and poor outcomes. ${ }^{1}$ Mortality among patients with CKD and ESRD remains high due to its association with the occurrence of ischemic heart disease (IHD). ${ }^{2}$ Numerous data show a close relationship between inflammation and cardiovascular morbidity and mortality. Hs-CRP is an important indicator and diagnostic tool for systemic inflammation. It has been found that
Interleukin- 6 and tumour necrosis factor- $\alpha$ are proinflammatory cytokines that can stimulate CRP synthesis in the liver. ${ }^{3}$ All these biomarkers are very useful in the prediction of cardiovascular complications and mortality in ESRD patients. ${ }^{4}$ Renal function impairment frequently leads to IHD. There are many traditional risk factors associated with IHD. Nowadays non-traditional CKD-specific risk factors for IHD appeared. Among the most important non-conventional risk factors are uremic toxins (UTs). Despite many studies either 
experimental or clinical that showed the relation between the accumulation of UTs and IHD progression, these relationships are not fully understood until now. UTs were suggested to have a principal role in the CKD and IHD progression. ${ }^{5}$ One of these UTs is asymmetric dimethylarginine (ADMA) and its symmetric enantiomer, symmetric dimethylarginine (SDMA), both of them inhibits the enzyme Nitric Oxide (NO) synthase that acts as a regulator for NO synthesis, It has been found that ADMA and SDMA have close relation with atherosclerosis, cardiovascular and renal diseases. ${ }^{6}$ Also, ADMA has been suggested to have a role in the pathogenesis of different conditions such as Alzheimer's disease, the resistance of insulin, dysfunction of the thyroid gland, erectile dysfunction, and even infertility. ${ }^{7}$ Although plasma levels ADMA are elevated in renal function impairment, this elevation is not only due to decreased renal clearance of ADMA but also it has been reported that more than $90 \%$ of the metabolism of ADMA is by two enzymes dimethylarginine dimethylaminohydrolase I (DDAH)-I and II, both of these enzymes are highly expressed in the kidney. ${ }^{8}$ CKD has been found to decrease the expression of DDAH, which has a major contribution to the elevation of plasma ADMA levels caused by decreased clearance by these two enzymes. ${ }^{9}$ It is well known that decreasing the concentration of NO by ADMA not only affects blood pressure but also acts as a regulator of Glomerular filtration barrier and is associated with permeability of albumin so it worsens the kidney condition. ${ }^{10}$

It has been found that increasing ADMA leads to decrease NO through inhibition of NO synthase. NO has a major effect on the cardiovascular system so decrease NO production by ADMA has a major role in atherosclerosis and IHD. ${ }^{11}$

Not only NO is known as a risk factor of atherosclerosis but also oxidized low-density lipoprotein (ox-LDL) can promote atherosclerosis progression. ${ }^{12}$

It has been recognized that LOX-1 takes part in the development of atherosclerosis ${ }^{21}$ through interaction between LOX-1 and ox-LDL which leads to induction of dysfunction of endothelium, adhesion of leukocyte, formation of macrophage-derived foam cell, proliferation and migration of smooth muscle cell, and activation of platelet ${ }^{13}$, and inhibited LOX1 expression could block the pathogenesis progression of atherosclerosis. ${ }^{14}$

Activation of LOX-I eventually causes atherosclerotic plaques rupture and acute cardiovascular sequences. Moreover, cleavage of LOX-1 generates soluble LOX-1 (sLOX-1), which is considered one of the most useful diagnostic and prognostic markers for atherosclerosis-related diseases in human patients. For medication, many natural products and drugs used clinically have appeared as LOX-I inhibitors then have been discovered to have antiatherosclerosis effects. ${ }^{15}$

So, this study aimed to assess the clinical utility of ADMA and LOX-1 in addition to hs-CRP as biomarkers of IHD in ESRD patients with regular hemodialysis.

\section{PATIENTS AND METHODS}

This study was conducted at Al-Azhar University Hospitals from May 2018 to March 2019 and included 59 patients with CKD. (Group I). They were subdivided to: Subgroup Ia $(\mathrm{n}=27)$, they were subjected for regular dialysis and having IHD as evidenced by history taking for previous IHD, ECG and echocardiography, Subgroup Ib $(n=13)$, they were subjected for regular dialysis and had no history suggestive of IHD and Subgroup Ic $(n=19)$, they had CKD, at different stages and they are on conservative treatment. Controls (group II): This group included 29 apparently healthy subjects they were matched in age and sex.

Exclusion criteria include chronic liver diseases, autoimmune diseases, thyroid dysfunction, lupus nephritis, diabetes mellitus, marked obesity, severe malnutrition, and irregular hemodialysis patients. Approval of the local Ethics and research Committee and obtaining written informed consent were performed.

All participants were subjected to full medical history taking, full clinical examination, and twelve leads resting ECG, Echocardiography, and the following Laboratory investigations:

Sampling: Eight $\mathrm{ml}$ of venous blood was withdrawn from all participants under complete aseptic conditions and used as follows: $1 \mathrm{ml}$ on EDTA for complete blood count, $1 \mathrm{ml}$ on Fluoride for assay of random blood sugar. $6 \mathrm{ml}$ were left at room temperature to be clotted then centrifuged at 3000 rpm for 10 minutes to separate serum which was divided into two separate aliquots. One aliquot used for routine biochemical laboratory tests (ALT, AST, Urea, Creatinine, Uric acid, Total cholesterol, Triglycerides, and HDL cholesterol). The other aliquot was stored at $-20{ }^{\circ} \mathrm{C}$ until used for assay of serum ADMA, LOX-1, and hs-CRP.

Laboratory investigations include

CBC determined by Sysmex KX-21N which is an automated cell counter (TAO Medical Incorporation, Japan), renal function tests, liver function tests, random blood sugar, and lipid profile were assayed by fully automated clinical chemistry analyzer Cobas c311 (Roche Diagnostics), high sensitivity Creactive protein concentration determination by commercially available ELISA Kit supplied by DRG International Inc., Quantitative determination of serum ADMA by ELISA technique. by commercially available ELISA Kit supplied by BT LAB Bioassay Technology Laboratory, and Quantitative determination of serum LOX-1 by ELISA technique using commercially available Human LOX-1 ELISA Kit (ab212161) abcam. Analyzer used: Stat fax- 2600 (Chro Mate) Awareness Technology, Inc Statistical Analysis

The program used in statistical methods was SPSS version 25.0 (Chicago IL, USA).

The diagnostic performance and detecting best cut off values were evaluated using the ROC curve. 


\section{RESULTS}

Results of this study are shown in (Tables 1-6) and (Figures 1-3).

Descriptive statistics of different studied parameters in all studied groups are shown in Table 1

\begin{tabular}{|l|c|c|c|c|}
\hline \multicolumn{1}{|c|}{ Characteristics } & $\begin{array}{c}\text { Subgroup Ia } \\
(\mathbf{n = 2 7})\end{array}$ & $\begin{array}{c}\text { Subgroup Ib } \\
(\mathbf{n = 1 3})\end{array}$ & $\begin{array}{c}\text { Subgroup Ic } \\
(\mathbf{n = 1 9})\end{array}$ & $\begin{array}{c}\text { Control group } \\
(\mathbf{n}=\mathbf{2 9})\end{array}$ \\
\hline Age (year) & $43.9 \pm 13.8$ & $38.3 \pm 9.5$ & $47.6 \pm 5.7$ & $43.7 \pm 9.6$ \\
\hline Sex(male/female & $17 / 10$ & $9 / 4$ & $13 / 6$ & $19 / 10$ \\
\hline $\begin{array}{l}\text { Smoking history } \\
\text { (number/percent) }\end{array}$ & $7 / 25.9$ & $2 / 15.5$ & $6 / 31.6$ & $3 / 10.3$ \\
\hline Creat(mg/dL) & $8.82 \pm 0.71$ & $8.49 \pm 0.78$ & $2.21 \pm 0.52$ & $0.91 \pm 0.16$ \\
\hline BUN (mg/dl) & $128.37 \pm 22.68$ & $100 \pm 16.25$ & $72.63 \pm 16.49$ & $13.21 \pm 4.26$ \\
\hline Uric acid mg/dL & $6.08 \pm 0.82$ & $5.85 \pm 1.02$ & $5.22 \pm 1.07$ & $4.53 \pm 0.75$ \\
\hline Total Cholesterol (mg/dl) & $200 \pm 24.5$ & $190.08 \pm 22.6$ & $187.89 \pm 14.7$ & $174.48 \pm 10.4$ \\
\hline TG mg/dl9mg/dL) & $148.89 \pm 5.12$ & $141 \pm 12.6$ & $110.95 \pm 10.6$ & $125.2 \pm 11.0$ \\
\hline HDL - Cholesterol mg/dL & $45.33 \pm 5.03$ & $44.3 \pm 5.4$ & $54.6 \pm 6.1$ & $57.6 \pm 7.22$ \\
\hline hs-CRP *(mg/L) & $21(9-81) *$ & $19(12-34) *$ & $7(3-9) *$ & $3.8(1-6) *$ \\
\hline LOX-1(ng/L) & $4.4 \pm 1.7$ & $3.2 \pm 0.38$ & $2.31 \pm 0.34$ & $1.62 \pm 0.87$ \\
\hline ADMA (ng/L) & $29.7 \pm 8.4$ & $25.31 \pm 8.66$ & $25.26 \pm 5.8$ & $13.3 \pm 2.8$ \\
\hline
\end{tabular}

Table 1: Baseline clinical and laboratory characteristics of the studied groups Data are presented as mean \pm SD or median and range for skewed data*

As shown in Table 2, regarding the level of hs-CRP in subgroups $\mathrm{Ia}, \mathrm{Ib}$, and Ic there is a significantly higher level in subgroup Ia when compared with subgroup $\mathrm{Ib}(\mathrm{p}=0.025)$ also there was a significant increase in subgroup $\mathrm{Ib}$ when compared with subgroup Ic meanwhile studying of the difference between subgroup Ia and subgroup Ic there was a highly significant difference with p-value $<0.000$.

As regards the LOX-1 in different subgroups there was a highly significant difference when comparing every two subgroups with the highest values in subgroup Ia.

Also, Table 2 shows the comparison of serum ADMA level in different subgroups showing that no significant difference between subgroup Ia and subgroup Ib also no difference between subgroup Ib and Ic while the comparisons between subgroup Ia and subgroup Ic show a significant difference with $\mathrm{p}$-value $=0.014$.

\begin{tabular}{|l|c|c|c|c|c|c|}
\hline \multicolumn{1}{r|}{ Parameter } & \multicolumn{2}{c}{ Subgroup Ia/ Ib } & \multicolumn{2}{c|}{ Subgroup Ia/ Ic } & \multicolumn{2}{c|}{ Subgroup Ib/ Ic } \\
\cline { 2 - 8 } & $\mathrm{t} / \mathrm{z}^{*}$ & $\mathrm{P}$ & $\mathrm{t} / \mathrm{z}^{*}$ & $\mathrm{P}$ & $\mathrm{t} / \mathrm{z}^{*}$ & $\mathrm{P}$ \\
\hline hs-CRP (mg/L) & $0.849^{*}$ & 0.025 & $5.662^{*}$ & .000 & $6.98^{*}$ & .000 \\
\hline LOX -I (ng/L) & 3.422 & 0.002 & 6.076 & .000 & 6.909 & .000 \\
\hline ADMA (ng/L) & 1.53 & 0.134 & 2.104 & 0.014 & 0.017 & 0.986 \\
\hline
\end{tabular}

Table 2: Statistical comparison between the two subgroups in the patient's group

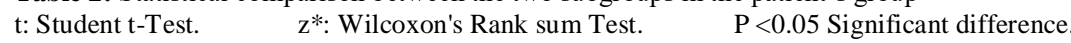


As shown in Table 3 there were significantly higher levels of serum hs-CRP, serum LOX-1, and serum ADMA in subgroups Ia, Ib, and Ic when compared with the control group with P-values $<0.000$.

\begin{tabular}{|l|c|c|c|c|c|c|}
\hline \multicolumn{1}{r|}{ Parameter } & \multicolumn{2}{c}{ Subgroup Ia/ II } & \multicolumn{2}{c|}{ Subgroup Ib/II } & \multicolumn{2}{c|}{ Subgroup Ic/ II } \\
\cline { 2 - 8 } & $\mathrm{t} / \mathrm{z}^{*}$ & $\mathrm{P}$ & $\mathrm{t} / \mathrm{z}^{*}$ & $\mathrm{p}$ & $\mathrm{t} / \mathrm{z}^{*}$ & $\mathrm{p}$ \\
\hline hs-CRP (mg/L) & $6.716^{*}$ & .000 & $8.677^{*}$ & .000 & $8.35^{*}$ & .000 \\
\hline LOX -1(ng/L) & 7.459 & .000 & 8.122 & .000 & 3.809 & .002 \\
\hline ADMA (ng/L) & 9.575 & .000 & 4.861 & .000 & 8.226 & .000 \\
\hline
\end{tabular}

Table 3: Comparison between different subgroups in the patient's group versus the control group in different studied parameters t: Student t-Test. $\quad \mathrm{z} *$ : Wilcoxon's Rank sum Test. $\quad \mathrm{P}<0.05$ Significant difference.

Table 4 shows the correlations between hs-CRP, LOX-1, and ADMA with other different parameters in subgroup Ia (ESRD with IHD). hs-CRP showed a statistically significant positive correlation with serum ADMA and serum LOX-1 while the other studied parameters show a non-statistically significant correlation with hs-CRP (serum Creatinine, Blood Urea, Total Cholesterol, TG, HDL, and serum Uric acid).

\begin{tabular}{|c|c|c|c|c|c|c|}
\hline \multirow[t]{2}{*}{ Parameter } & \multicolumn{2}{|c|}{ hs-CRP } & \multicolumn{2}{|c|}{ ADMA } & \multicolumn{2}{|c|}{ LOX -1 } \\
\hline & $\mathrm{R}$ & $\mathrm{P}$ & $\mathrm{r}$ & $\mathrm{p}$ & $\mathrm{R}$ & $\mathrm{p}$ \\
\hline BUN (mg/dl) & 0.093 & 0.646 & $-0.014-$ & 0.943 & $-0.162-$ & 0.419 \\
\hline CREAT (mg/dl) & -0.084 & 0.667 & $-0.057-$ & 0.778 & $-0.378-$ & 0.052 \\
\hline UA (mg/dl) & 0.081 & 0.688 & 0.324 & 0.1 & 0.032 & 0.873 \\
\hline CHOLES (mg/dl) & 0.060 & 0.765 & $-0.082-$ & 0.686 & 0.147 & 0.466 \\
\hline TG (mg/dl) & 0.344 & 0.039 & $-0.392-^{*}$ & 0.043 & $-0.027-$ & 0.893 \\
\hline HDL (mg/dl) & 0.273 & 0.168 & 0.206 & 0.303 & 0.101 & 0.615 \\
\hline CRP (mg/L) & 1.000 & --- & $0.644^{*}$ & $0.000^{*}$ & 0.421 & 0.043 \\
\hline ADMA (ng/L) & $0.644^{*}$ & $0.000 *$ & 1.000 & ---- & $0.391 *$ & $0.040^{*}$ \\
\hline LOX-1 (ng/L) & 0.421 & 0.043 & $0.391 *$ & $0.040^{*}$ & 1.000 & ---- \\
\hline
\end{tabular}

Table 4: Correlation studies between serum hs-CRP, serum ADMA, and Serum LOX-1 in ESRD subgroup with IHD (subgroup Ia)

$\mathrm{P}>0.05$ : Non-significant correlation $\quad \mathrm{P}<0.05$ : Significant correlation

The receiver operating characteristics (ROC) curve was applied to detect the diagnostic performance of hs-CRP, LOX-1, and ADMA for differentiating ESRD with IHD from ESRD patients without IHD as shown from Figures 1-3 and Table 5.

\begin{tabular}{|c|c|c|c|c|c|c|c|c|c|}
\hline & & + & - & Total & PPV & NPV & Sensitivity & Specificity & $\begin{array}{c}\text { Overall } \\
\text { Diagnostic } \\
\text { Efficacy }\end{array}$ \\
\hline \multirow[t]{3}{*}{ CRP } & ESRD with IHD & 5 & 22 & 27 & \multirow{3}{*}{$100 \%$} & \multirow{3}{*}{$37.14 \%$} & \multirow{3}{*}{$18.5 \%$} & \multirow{3}{*}{$100 \%$} & \multirow{3}{*}{$45 \%$} \\
\hline & ESRD without IHD & 0 & 13 & 13 & & & & & \\
\hline & Total & 5 & 35 & 40 & & & & & \\
\hline \multirow[t]{3}{*}{ ADMA } & ESRD with IHD & 19 & 8 & 27 & \multirow{3}{*}{$82.61 \%$} & \multirow{3}{*}{$52.94 \%$} & \multirow{3}{*}{$70.4 \%$} & \multirow{3}{*}{$69.2 \%$} & \multirow{3}{*}{$70 \%$} \\
\hline & ESRD without IHD & 4 & 9 & 13 & & & & & \\
\hline & Total & 23 & 17 & 40 & & & & & \\
\hline LOX-1 & ESRD with IHD & 12 & 15 & 27 & $100 \%$ & $46.43 \%$ & $44.4 \%$ & $100 \%$ & $62.5 \%$ \\
\hline
\end{tabular}

Table 5: Diagnostic accuracy at the optimal cut off value of each biomarker 

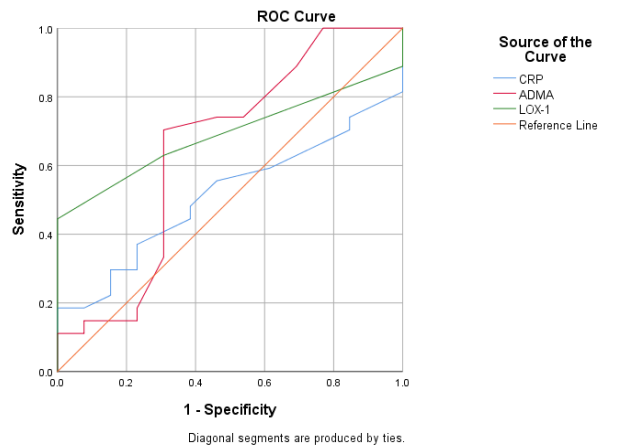

\section{The area under the ROC curves}

\begin{tabular}{|l|l|}
\hline CRP & $51.3 \%$ \\
\hline ADMA & $64.8 \%$ \\
\hline LOX-I & $69.1 \%$ \\
\hline
\end{tabular}

Fig. 1: ROC curve showing diagnostic performance of the biomarkers discriminating patients with ESRD + IHD from ESDR patients without IHD

Diagnostic accuracy of the combined CRP + LOX1 in discriminating patients with ESRD+IHD from ESDR patients without IH. Area under the ROC curve, sensitivity, and specificity of the combined CRP+LOX1

\section{ROC curve}

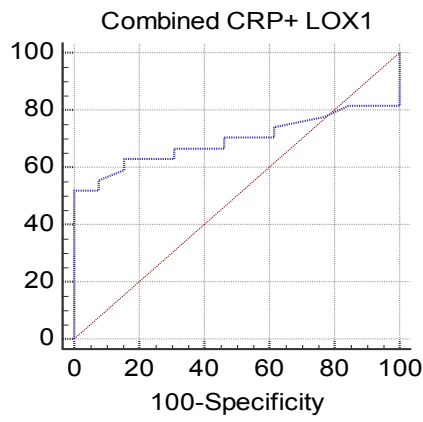

\begin{tabular}{|c|c|}
\hline \begin{tabular}{|c|} 
The area under the \\
ROC curve (AUC) \\
Sensitivity
\end{tabular} & $\mathbf{0 . 6 9 5}$ \\
\hline Specificity & $100 \%$ \\
\hline Standard Error $^{\mathrm{a}}$ & 0.0828 \\
\hline 95\% Confidence interval & 0.530 to 0.831 \\
\hline z statistic & 2.356 \\
\hline Significance level P (Area $=0.5)$ & 0.0185 \\
\hline
\end{tabular}

Fig. 2: ROC curve showing diagnostic accuracy of the combined CRP + LOX1 in discriminating patients with ESRD+IHD from ESDR patients without IHD

Diagnostic accuracy of the combined CRP+ADMA in discriminating patients with ESRD+IHD from ESDR patients without IHD

\section{ROC curve}

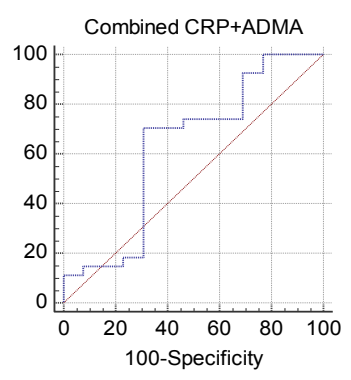

\begin{tabular}{|c|c|}
\hline $\begin{array}{l}\text { The area under } \\
\text { the ROC curve (AUC) }\end{array}$ & 0.627 \\
\hline Sensitivity & $70.37 \%$ \\
\hline Specificity & $69.23 \%$ \\
\hline Standard Error ${ }^{\mathrm{a}}$ & 0.107 \\
\hline $95 \%$ Confidence interval $^{\mathrm{b}}$ & 0.46 to 0.774 \\
\hline z statistic & 1.183 \\
\hline $\begin{array}{l}\text { Significance level } \\
\mathrm{P}(\text { Area }=0.5)\end{array}$ & 0.2369 \\
\hline
\end{tabular}

Fig. 3: ROC curve showing diagnostic accuracy of the combined CRP+ADMA in discriminating patients with ESRD+IHD from ESDR patients without IHD 
Comparison of the diagnostic accuracy of all biomarkers in discriminating patients with ESRD+IHD from ESDR patients without IHD

\begin{tabular}{|c|c|c|c|}
\hline & AUC & $\mathbf{S E}^{\mathrm{a}}$ & $95 \% \mathrm{CI}^{\mathrm{b}}$ \\
\hline CRP & 0.513 & 0.0919 & 0.350 to 0.674 \\
\hline LOX1 & 0.691 & 0.0769 & 0.525 to 0.827 \\
\hline ADMA & 0.648 & 0.106 & 0.481 to 0.792 \\
\hline$\underline{\text { Combined CRP+LOX1 }}$ & $\underline{0.695}$ & $\underline{0.0828}$ & $\underline{0.530 \text { to } 0.831}$ \\
\hline Combined CRP+ADMA & 0.627 & 0.107 & 0.460 to 0.774 \\
\hline
\end{tabular}

* Table 6: The largest AUROC is underlined.

\section{DISCUSSION}

Chronic kidney disease (CKD) is one of the main causes of morbidity and mortality. Ischemic heart disease (IHD) and bone mineral disorder (BMD) are the principal causes of death in patients with end-stage renal disease (ESRD). ${ }^{16}$

It is well known that the identification of CKD patients at stages G1 and G2 and the implementation of therapeutic strategies lead to improving the prognosis of those patients, reducing the incidence of ischemic events and ESRD. ${ }^{17}$

So, the present study aimed to assess the clinical significance of LOX-1 and ADMA as markers of IHD in ERSD patients subjected to hemodialysis.

Oxidized LDL (ox-LDL) is a major contributor in the process of atherosclerosis by affecting many cells such as endothelial cells, smooth muscle cells macrophages, platelets, and fibroblasts via its interaction with LOX-1 receptor. ${ }^{18}$

Multiple lines of evidence have demonstrated that activation of endothelial LOX-1 results in endothelial dysfunction, characterized by reduced vasodilation, proinflammatory state, and prothrombotic properties. Activation of LOX-1 with oxLDL induces cell injury and apoptosis in human coronary artery endothelial cells. ${ }^{19}$

Asymmetric dimethylarginine (ADMA) and its enantiomer, symmetric dimethylarginine (SDMA), both are natural amino acids intracellularly generated. ADMA makes this effect in IHD as it is one of the most potent endogenous inhibitors of nitric oxide synthase (NOS) and, therefore, inhibits the nitric oxide production (NO), Which play a key role in the regulation of vascular tone. ${ }^{20}$

Sibal L et al ${ }^{21}$ stated that ADMA has been found as an independent risk factor for $\mathrm{IHD}$ and has a key role in the proatherogenic activities, Koenig W, confirmed the role of inflammation in the propagation of atherosclerosis. So, one of the most important inflammatory biomarkers, hs-CRP has gained the most focus for its usage as a screening tool, risk reclassification as well as its role in the prediction of Statin therapy clinical response. ${ }^{22}$

Our results showed that hs-CRP was significantly higher in ESRD patients (subgroup Ia and Ib) when compared with CKD patients on conservative treatment (subgroup Ic) as well as control group (group II) this results came in agreement with many studies as Rahma Nefzi et al., ${ }^{23}$

Moreover, the results of this work revealed a significant increase in serum hs-CRP in ESRD patients with IHD (subgroup Ia) when compared with these patients of ESRD without IHD (subgroup Ib). These results coincide with ElSenosy FM and his colleagues. ${ }^{24}$

Regarding LOX-1 and ADMA, a significant increase was detected in ESRD patients with IHD (subgroup Ia) when compared with CKD patients on conservative treatment (subgroup Ic).

Moreover, comparing serum LOX-1 and ADMA in different subgroups with the control group (group 2) showed a statistically significant difference with higher levels in patient subgroups than the control group. This finding came in accordance with Dogan I et al., who stated that the increased serum LOX-1 in uremic patients is a constant finding. ${ }^{25}$

Also, our findings came in agreement with Rashed et al., who reported that chronic renal failure and ESRD patients on regular hemodialysis have significantly increased in ADMA than do control subjects. $^{26}$

UgurcuV and his colleagues explained the significant increase of serum ADMA in patients on hemodialysis and chronic renal failure stage- 4 by the higher levels of methylation of protein, increased rate of protein turnover moreover increased ADMA is due to its depressed excretion and decreased activity of DDAH enzymes that are used in its catabolism. ${ }^{27}$

Also, results obtained in this study went hand by hand with the results reported by Böger and Schwedhelm who found that plasma ADMA levels were significantly higher in patients with heart diseases, and their finding clarifies the role of ADMA in the pathophysiology of IHD. ${ }^{28}$

Kones R stated that a unified cut point for hs-CRP based on a single value should not be applied universally among individuals. The interpretation to that was many reasons as hs- CRP genetic polymorphism, the effect of genetic loci that affects CRP response, also, lifestyle, ethnic, and gender that causes variation in concentration of hs-CRP, moreover body mass index (BMI), metabolic syndrome, DM cause remarkable variation of its baseline. ${ }^{29}$

Concerning correlation studies, this study showed statistically significant positive correlations between hs- CRP, LOX-1, and ADMA in subgroup Ia (ESRD with IHD).

In assessing the diagnostic performance of the studied markers in ESRD with IHD patients (subgroup Ia) versus ESRD without IHD patients (subgroup Ib), we found that hs-CRP had a superior diagnostic performance when compared with the two other biomarkers. From our results, the largest AUC in ROC was detected for the combined use of hs-CRP with LOX-1.

Therefore, the combined assessment of serum hs- CRP and LOX-1 resulted in an improvement in the performance and raising the chance of differentiating ESRD with IHD form those without IHD. So it may be more helpful to add LOX-1, as known biomarkers with proven roles in atherosclerosis, to the famous inflammatory marker, hs-CRP, with well-known variability among individuals by many factors, especially when assessing the cardiovascular disease risk in ERSD patients subjected to hemodialysis.

\section{CONCLUSION}

LOX-1 and ADMA are uremic toxins, LOX-1 is more reliable than ADMA as s potential biomarker for the detection of ESRD patients who are at a high risk of IHD and the effectiveness of this biomarker is increased when it used in combination with other proven markers as hs-CRP.

\section{REFERENCES}


1. Gravis J. Diagnosis and management of chronic kidney disease. Mayo Clin. Proc.2008; 83(9):1064

2. Niizuma S, Iwanaga $\mathrm{Y}$, Yahata $\mathrm{T}$ et al. Renocardiovascular Biomarkers: from the Perspective of Managing Chronic Kidney Disease and Cardiovascular Disease. Front. Cardiovasc. Med. 2017; $4: 10$

3. Stenvinkel P, Barany P, Heimbürger $\mathrm{O}$ et al. Mortality, malnutrition, and atherosclerosis in ESRD: what is the role of interleukin 6? Kidney Int Suppl 2002; 80:103-8.

4. Roberts M, Srivastava P, Macmillan N, et al. B-type natriuretic peptides strongly predict mortality in patients who are treated with long-term dialysis. Clin J Am Soc Nephrol 2008; 3:1057-65.

5. Fujii H, Goto S, and Fukagawa M. Role of uremic toxins for kidney, cardiovascular, and bone dysfunction. Toxins (Basel) 2018; 10(5): 202.

6. Vanholder R, Baurmeister U, Brunet P, et al. A bench to bedside view of uremic toxins. J Am Soc Nephrol. 2008;19: 863-870.

7. Leiper J, Santa Maria J, Chubb A et al. Identification of two human dimethylarginine dimethylaminohydrolase with distinct tissue distributions and homology with microbial arginine diminishes. Biochem. J. 1999; 343:209-214.

8. Sharma M, Zhou Z, Miura H, et al. ADMA injures the glomerular filtration barrier: Role of nitric oxide and superoxide. Am. J. Physiol. Ren. Physiol.2009;296: F1386-F1395.

9. Cooke $\mathrm{J}$ and Tsao P. Is NO an endogenous antiatherogenic molecule? Arterioscler. Thromb. (1994); 14:653-655.

10. Witztum J and Steinberg D. Role of oxidized low-density lipoprotein in atherogenesis. J. Clin. Investig 1991; 88:1785-1792.

11. Kume N, Murase T, Moriwaki H, et al. Inducible expression of lectin-like oxidized LDL receptor-1 in vascular endothelial cells. Circ Res 1998; 83:322-327

12. Kunming T, Sayoko O, Peter J. et al. LOX-1 in atherosclerosis and vasculopathy: current knowledge and future perspectives Ann. N.Y. Acad. Sci. 1443 (2019) 34-53C

13. National Kidney Foundation. Improving Global Outcomes (KDIGO) CKD Work Group. KDIGO 2012 Clinical Practice Guideline for the Evaluation and Management of Chronic Kidney Disease. Kidney Int Suppl. 2013; 3:4-150.

14. Foley R, Parfrey P, and Sarnak M. Clinical epidemiology of the cardiovascular disease in chronic renal disease. Am J Kidney Dis 1998; 32: S112-9.

15. Nephrology, J. S. O. Evidence-based Clinical Practice Guideline for CKD 2013 346-423 (2014).
16. Qaseem, A. Screening, monitoring, and treatment of stage 1 to 3 chronic kidney disease: A clinical practice guideline from the American College of Physicians. Ann Intern Med 2013;159, 835847.

17. Ajoe J, Sri H and Jawahar L. Role of Ox-LDL and LOX-1 in Atherogenesis. Current Medicinal Chemistry 2019; 26: 1693.

18. Xu S, Ogura S, Chen J et al. LOX-1 in atherosclerosis: biological functions and pharmacological modifiers. Cellular and molecular life sciences: CMLS 2013 vol. 70, 16; 2859-72.

19. Li D, Chen H, Staples E, et al. Oxidized low-density lipoprotein receptor LOX-1 and apoptosis in human atherosclerotic lesions. $J$ Cardiovasc Pharmacol Ther. 2002; 7:147-53.

20. Leone A, Moncada S, Vallance P, et al. Accumulation of an endogenous inhibitor of nitric oxide synthesis in chronic renal failure. Lancet. 1992; 339:572-575.

21. Sibal L, Agarwal S, Home $P$ et al. The Role of asymmetric Dimethylarginine (ADMA) in endothelial dysfunction and cardiovascular disease. Curr Cardiol Rev. 2010;6(2):82-90.

22. Koenig W. High-sensitivity C-reactive protein and atherosclerotic disease: from improved risk prediction to risk-guided therapy. Int $J$ Cardiol. 2013; 168(6):5126-34

23. Rahma N, Amine L, Ben Ammar H et al. T94. Hs-CRP to evaluate chronic inflammation and cardiovascular diseases in Schizophrenia Schizophr Bull. 2018; 44(Suppl 1): S152.

24. El Senosy F, Morsy M, Mohamed N, et al. Evaluation of serum YKL-40 and cardiovascular risk in chronic kidney disease. Sci J AlAzhar Med Fac Girls 2018; 2:64-74

25. Dogan I, Dogan T, Yetim M, et al. Relation of serum ADMA, Apelin-13, and LOX-1 levels with inflammatory and echocardiographic parameters in hemodialysis patients. Ther Apher Dial 2018; 22(2):109-117

26. Rashed A, Soliman K, Aun A, et al. Relationship between asymmetric Dimethylarginine (ADMA) and atherosclerosis in chronic renal failure patients. J Nephrol Ther 2017; 7: 300.

27. Ugurcu V, Vatansev H, Unlu A, et al. Levels of arginine and its products in dialysis patients. Eur Rev Med Pharmacol Sci .2014; 18: 2357-2364.

28. Schwedhelm E and Böger R. The role of asymmetric and symmetric dimethylarginines in renal disease. Nat Rev Nephrol 2011 ; 7: 275285 .

29. Kones R. Rosuvastatin, inflammation, C-reactive protein, JUPITER, and primary prevention of cardiovascular disease, a perspective study. Drug Des Devel Ther, 2010, pp. 383-413 